\title{
Screening, Prevention and Treatment of COVID-19 with Special
}

\section{Reference to African-Americans}

\author{
Anita Mandal ${ }^{1}$, Noor Islam ${ }^{2}$, Benjamin Okafor ${ }^{3}$ and Prabir Mandal ${ }^{4 *}$ \\ ${ }^{1}$ Department of General Studies, Edward Waters College, Florida, USA. \\ ${ }^{2}$ Department of Social \& Behavioral Sciences, Edward Waters College, Florida, USA. \\ ${ }^{3}$ Department of Criminal Justice \& Forensic Sciences, Edward Waters College, Florida, USA. \\ ${ }^{4}$ Mathematics \& Sciences, Edward Waters College, 1740 Kings Road, Jacksonville, FL 32209. \\ *Corresponding Author: Prabir K. Mandal. Department of Mathematics \& Sciences, Edward Waters College, 1658 Kings Road, Jacksonville, \\ FL 32209.
}

Received date: February 20, 2021; Accepted date: March 02, 2021; Published date: March 08,2021

Citation: Mandal A., Islam N., Okafor B. and Prabir K. Mandal, (2021) Screening, Prevention and Treatment of COVID-19 with Special Reference to African-Americans J, Biotechnology and Bioprocessing 2(3); DOI: 10.31579/2766-2314/029

Copyright: () 2021, Prabir K. Mandal This is an open access article distributed under the Creative Commons Attribution License, which permits unrestricted use, distribution, and reproduction in any medium, provided the original work is properly cited.

\begin{abstract}
Viruses mutate all the time, and the coronavirus that causes COVID-19 is no exception. As new variants of the COVID-19 emerge, a slew of new studies suggest that some may be able to evade immune responses triggered by a previous infection or by a vaccine. The novel variants of SARS-CoV-2 including those seen in the UK (2OI/5O1Y.V1/B.1.1.7), South Africa (2OH/5O1Y.V2/B.1.351), Brazil (P.1/2OJ/5O1Y.V3/B.1.1.248) have emerged with the concern of increased infectivity and virulence. Scientists are working to learn more about the characteristics of these strains whether they could cause more severe illness, and whether currently authorized vaccines will protect people against them. All viruses naturally mutate and evolve over time. For example, flu virus change often, which is why doctors recommend that you get a flu shot every year. Various mRNA vaccine platforms have been developed in recent years and validated in studies of immunogenicity and efficacy.

Keywords: CDC; COVID-19; SARS-CoV-2; Vaccine; African-Americans; mRNA; USFDA
\end{abstract}

\section{Introduction}

In the last 20 years, there have been three coronavirus infections. They are severe acute respiratory syndrome coronavirus (SARS-CoV), Middle East respiratory syndrome coronavirus (MERS-CoV) and SARS-CoV-2. In early 2020, a new virus began generating headlines all over the world because of the unprecedented speed of its transmission. The disease caused by an infection with SARS-CoV-2 is called COVID-19 (caused by SARS-CoV-2 infection), which stands for coronavirus disease 2019. COVID-19 is a major cause of death and disability worldwide, totaling 89.4 million cases and 1.9 million deaths globally as of January 9, 2021 Coronaviruses are named for the crown-like spikes on the surfaces. Viruses constantly change through mutation, and new strains of a virus are expected to occur. Multiple strains of the virus that causes COVID-19 have been documented. There are about 23 genetic changes in this strain. In the United Kingdom (U.K.), a new but more transmissible variant called B.1.1.7 has emerged in September 2020 with an unusually large number of mutations. In South Africa, another variant called B.1.351 has emerged independently of the variant detected in the U.K. In Brazil, a variant called P.1 emerged, which contains a set of additional mutations that may affect its ability to be recognized by antibodies. The new strains also appeared in Denmark, the Netherlands, and other European countries. 54 cases of the U.K. variant had been identified in the U.S. Currently, there is no evidence that these strains cause more severe illness or increased risk of death, but it spreads up to $70 \%$ more easily. The U.K. variant has been detected in nine U.S. states viz., California, Colorado, Connecticut, Florida, Georgia, New York, Ohio, Oregon and Pennsylvania. Helix and Illumina (commercial testing companies) partnered with U.S. Centers for Disease Control and Prevention (CDC) screened more than 2 million COVID tests for signs of the variant. They forwarded 158 samples for further testing and found 51 were positive for the U.K. variant. The U.K. and South African variant have the same mutation on their spike protein called N501Y. The spike proteins on the virus rotate between open and closed positions. They can attach to doors on our cells called ACE2 receptors only when they are in their open positions.

Black Americans are more likely to be diagnosed, to be hospitalized, and to die from COVID-19. The death rate from COVID-19 has been reported to be 2-3 times higher among Black versus White individuals. Black individuals comprise $13.4 \%$ the U.S. population, yet account for more than 24\% of COVID-19 deaths (CDC, 2020; Bogart et al., 2021). The impact of COVID-19 goes well beyond the viral infection itself, reflecting the dynamics of a long-standing adverse host environment, including access to limited resources (i.e., money, food, education, health care, job flexibility), making disadvantaged communities more vulnerable in the pandemic (Ferdinand and Nasser, 2020). A recent report from the Pew Research Center indicated that three out of four African Americans in their study responded that religion was important to them and nearly half of these individuals (47\%) attended church weekly Bruce 2020). A study 
conducted by Block et al. (2020) reports that African Americans have lower than $80 \%$ adherence to four key COVID-19 public health recommendations - targeted education campaigns, consistent messaging across political groups, African American community leaders, and media outlets to improve compliance, in turn decreasing risk. Having higher incidence of hypertension, diabetes, malignancies, heart failure, cerebrovascular disease and underlying pulmonary/cardiovascular disease could make the African American community a potential high risk group for COVID-19 (Jahromi and Hamidianjahromi, 2020). Nauen et al. (2021) reported an atypical form of neurologic impairment by analysis of brain tissue from patients who died of COVID-19 that they had not seen megakaryocytes in brain vessels prior to this pandemic and found no reference in literature. The connection between high risk of African Americans for the COVID-19 virus and the higher incidence to chronic disease such as diabetes and cardiovascular disease may be associated with the inactivation of anti-aging genes that are critical to cell survival. In various chronic diseases these anti-aging genes are inactivated with altered immune responses. Activators of the anti-aging genes should be consumed to prevent these chronic diseases and the identification of inhibitor of the anti-aging genes should be assessed such as in the developing world where xenobiotics levels rise in the air, food and water (Ferdinand and Nasser, 2017; Mensah, 2018; Saab et al., 2015). The role of diet and lack of stress may be critical to improve the severity of COVID-19 and chronic diseases in African Americans.

\section{Screening/Tests}

There are two different types of tests - diagnostic and antibody. A diagnostic test can show if someone have an active coronavirus infection and should take steps to quarantine or isolate from others. Currently there are two types of diagnostic tests - molecular i.e., reverse transcriptasequantitative polymerase chain reaction (RT-qPCR) that detect the virus's genetic material and antigen tests that detect specific proteins from the virus. RT-qPCR assays being most commonly used. The technique uses liquids with the swab to extract the genetic material of the virus that may be on the swab. Then use special reagents (primers and probes), and a special machine to conduct several controlled heating and cooling cycles to convert the virus's RNA into DNA, and then make millions of copies of the DNA. When specific probes bind to DNA, a special type of light is produced that can be seen by a special machine which indicates the test is positive results for infection with SARS-CoV-2, the virus that causes COVID-19. CRISPR-based assays provide a robust and sensitive alternative for the detection of SARS-CoV-2 genomes. These assays use common and widely available reagents and are adaptable to minimal instrumentation and infrastructure (Rauch et al., 2021). An antibody test looks for antibodies that are made by immune system in response to a threat (a specific virus like COVID-19). The antibodies can take several days/weeks to develop after an infection.

\section{Treatments}

There is currently no cure for an infection with the new coronavirus. For milder illness, resting at home and taking medicine to reduce fever is often sufficient. The more severe cases require hospitalization, with treatment that might include supplemental oxygen, assisted ventilation and other supportive measures. In June 2020, preliminary results of an open-label randomized controlled trials (RCT) of dexamethasone showed that it significantly reduced critically ill COVID-19 patients receiving mechanical ventilation and, to a lesser degree, patients who need supplemental oxygen. In August 2020, plasma with antibodies from recovered COVID-19 patients (convalescent plasma therapy) showed both a prophylactic and therapeutic option. In October 2020, the U.S. Food and Drug Administration (USFDA) approved its first COVID-19 treatment with the medication, remdesivir (Veklury) as an intravenous (IV) infusion. Data from in vitro experimental studies indicated that hydroxychloroquine and chloroquine have an inhibitory effect on SARS-
CoV-2. Though Lopinavir/ritonavir were not effective for preventing intubation or death in moderately ill hospitalized patients with COVID19 , but treatment with tocilizumab was effective in reducing deaths and time spent in intensive care for critically ill patients with severe COVID19. In November 2020, under emergency use authorization (EUA) the USFDA granted monoclonal antibody medications e.g., bamlanivimab (Eli Lilly) and a combination of casirivimab-imdevimab (Regeneron Pharmaceuticals) administered via IV infusion were linked to decreased rates of hospitalization or visits to the emergency department. Colchicine (a common gout medication) and famotidine (the common antacid Pepcid) prevents COVID-19 hospitalizations. In November 2020, the USFDA also issued a EUA for the use of baricitinib in combination with remdesivir in hospitalized adults and children 2 years and older who require respiratory support. In February 2021, USFDA issued to Eli Lilly a EUA for bamlanivimab and etesevimab administered together for the treatment of mild to moderate COVID-19 in adults and pediatric patients who test positive for SARS-CoV-2 and who are at high risk for progressing to severe COVID-19.

\section{Vaccine}

Conventional vaccine approaches provide durable protection against a variety of dangerous diseases, but may not be applicable to non-infectious diseases, such as cancer. Therefore, the development of more potent and versatile vaccine platforms is urgently needed. In December 2020, the USFDA granted its first mRNA-based vaccines developed by PfizerBioNTech (BNT162b1) and Moderna (mRNA-1273). Pfizer and Moderna COVID-19 vaccines will work against the B.1.1.7 variant. If the messenger RNA (mRNA) vaccines end up being less effective against variants, they can quickly be reworked to target new sequences. That is a big advantage of that type of vaccine. One advantage of Moderna's mRNA technology is its flexibility, because it is based on the virus's genetic sequence. Developing a new vaccine against a new variant would be a matter of copying and pasting.

mRNA vaccines are a new type of vaccine to protect against infectious diseases. To trigger an immune response (to create antibodies), most vaccines put a weakened (or inactivated) germ (or disease causing pathogen) into our bodies. mRNA vaccines do not use the live virus that causes COVID-19, work by providing the genetic code for our cells to produce viral proteins. Using an appealing technique, the cells use DNA as the template to make mRNA molecules, which are then translated to build proteins. RNA vaccines work by introducing an mRNA sequence which is coded for a disease specific antigen. They do not affect or interact with our DNA in any way as mRNA never enters the nucleus of the cell, which is where our DNA is kept. When COVID-19 mRNA vaccines are inside the immune cells, the cells use them to make the spike protein piece, activating T-cells to fight infection and breaks down the mRNA (gets rid of them). There are issues to sort out, such as concerns about needing to store the vaccines in freezers-especially in the case of the Pfizer BioNTech vaccine, which needs to be kept at $-70^{\circ} \mathrm{C}$, whereas another vaccine developed by the German company CureVac, can be stored at $5^{\circ} \mathrm{C}$. Vaccines from DNA-based Oxford-AstraZeneca or Johnson \& Johnson need to be produced in lab-grown cells. Some scientists have suggested postponing the second shots of two-dose vaccines to make more available for people to get their first doses. The original recommended interval was 21 days between doses for the Pfizer vaccine and 28 days for the Moderna shots. Two-shot vaccinations aim for maximum benefit-the first dose primes immunological memory, and the second dose solidifies it. For both vaccines, a single dose protects about $50 \%$ (Pfizer) and 80\% (Moderna).

\section{Conclusion}

Vaccines alone won't be enough to bring the crisis under control. Rigorous and increased compliance with public health mitigation 
strategies, such as vaccination, physical distancing, use of masks, hand hygiene, good ventilation indoors, limiting gatherings of people in close proximity, isolation and self-quarantine, will be essential to limiting the spread of SARS-CoV-2 and protecting public health. High-income countries have reserved more than half of the world's COVID-19 vaccine doses despite representing just $14 \%$ of the world's population. Nationwide differences in the impact of COVID-19 could be explained, in part, by the national policies concerning the vaccination of children with BCG. However, clinical trials are underway to confirm this association.

As of February 3, 2021, 468,000 sequences of SARS-CoV-2 from COVID-19 cases globally have been uploaded into publicly available databases, including more than 93,000 from individuals in the U.S. The new variant could further exacerbate health disparities among communities of color. In comparison with DNA-based vaccines, mRNA vaccines can express target proteins more efficiently because of their expression in the cytoplasm without entering the nucleus. RNA based vaccines could have an impact due to their shorter manufacturing times and greater effectiveness. RNA vaccines have potential as novel therapeutic options for major diseases such as cancer. There is still a lot of work to be done before mRNA vaccines can become standard treatments. In the future, mRNA vaccine technology may allow for one vaccine to target multiple diseases. It is vitally important to continue research for the treatments to limit and cure COVID-19.

\section{References}

1. Block Jr R, Berg A, Lennon RP, Miller EL and Nunez-Smith M (2020) African American Adherence to COVID-19 Public Health Recommendations. Health Literacy Research and Practice, 4 (3): e166-e170.

2. Bogart LM, Ojikutu BO, Tyagi K, Klein DJ, Mutchler MG, Dong L, Lawrence SJ, Thomas DR and Kellman S (2021) COVID-19 Related Medical Mistrust, Health Impacts, and Potential Vaccine Hesitancy Among Black Americans Living With HIV. J Acquir Immune Defic Syndr 86 (2): 200-207.

3. Bruce MA (2020) Perspective: COVID-19 and African American Religious Institutions. Ethnicity \& Disease, 30 (3): 425-428.
4. Centers for Disease Control and Prevention (2020): COVID-19 in Racial and Ethnic Minority Groups. COVID-NET.

5. Ferdinand KC and Nasser SA (2017) Disparate Cardiovascular Disease Rates in African Americans: The Role of Stress Related to Self-Reported Racial Discrimination. Mayo Clin Proc, 92 (5): 689-692.

6. Ferdinand KC and Nasser SA (2020): African-American COVID19 Mortality. J Am Coll Cardiol, 75 (21): 2746-2748.

7. Jackson LA, Anderson EJ, Rouphael NG, Roberts PC, Makhene $\mathrm{M}$, et al (2020) An mRNA Vaccine against SARS-CoV-2Preliminary Report, N Engl J Med, 383: 1920-1931.

8. Jahromi AH and Hamidianjahromi A (2020) Why African Americans Are a Potential Target for COVID-19 Infection in the United States. J Med Internet Res, 22 (6): e19934.

9. Mensah GA (2018) Cardiovascular Diseases in African Americans: Fostering Community Partnerships to Stem the Tide. Am J Kidney Dis, 72 (5 Suppl 1): S37-S42.

10. Nauen DW, Hooper JE, Stewart CM and Solomon IH (2021) Assessing Brain Capillaries in Coronavirus Disease 2019. JAMA Neurology, E1-E2.

11. Pardi N, Hogan MJ, Porter FW and Weissman D (2018) mRNA vaccines- a new era in vaccinology. Nature Reviews, 17: 261-279.

12. Rauch JN, Valois E, Ponce-Rojas JC, Aralis Z, Lach RS, et al. (2021) Comparison of Seven Acute Respiratory Syndrome Coronavirus 2 Screening Using Reverse TranscriptaseQuantitative Polymerase Chain Reaction or CRISPR-Base Assays in Asymptomatic College Students. JAMA Network: 4 (2): e2037129.

13. Saab KR, Kendrick J, Yracheta JM, Lanaspa MA, Pollard M and Johnson RJ (2015) New Insights on the Risk for Cardiovascular Diasease in African Americans: The Role of Added Sugars. J Am Soc Nephrol, 26 (2): 247-257.

14. Xu S, Yang K, Li R and Zhang L (2020) mRNA Vaccine EraMechanisms, Drug Platform and Clinical Prospection. Int J Mol Sci, 21: 6582

15. Zhang W, Davis BD, Chen SS, Martinez JMS, Plummer JT and Vail E (2021) Emergence of a Novel SARS-CoV-2 Variant in Southern California. JAMA, E1-E3. 